\title{
Psicooncología
}

ISSN: $1696-7240$

http://dx.doi.org/10.5209/psic.71362

\section{Análisis situacional de los psicólogos de cuidados paliativos}

\author{
Leticia Ascencio Huertas ${ }^{1}$
}

Recibido: 31 de julio de 2020 / Aceptado: 29 de agosto de 2020

\begin{abstract}
Resumen: Introducción. Los pacientes con enfermedades crónicas en fase avanzada tienen numerosas necesidades psicológicas, sociales y espirituales, como resultado de su estado físico, condición, curso terapéutico y efectos secundarios del tratamiento, además de distrés psicológico y existencial por lo que la figura del psicólogo en Cuidados Paliativos tiene una gran relevancia. Objetivo. Realizar un análisis situacional de los psicólogos integrados a los equipos de cuidados paliativos del sector público en México. Método. Estudio prospectivo, descriptivo y transversal. Se diseño una encuesta ex profeso de 22 reactivos con respuesta dicotómica y opción múltiple. Resultados. Se analizaron un total de 49 encuestas de psicólogos que prestan sus servicios en unidades de atención médica en México, a través de análisis de frecuencias y porcentajes, chi cuadrada y w de wilcoxon. Conclusiones. Los psicólogos en cuidados paliativos requieren formación y capacitación formal para desarrollar habilidades y competencias específicas en este contexto; y de mayor sistematización y de protocolos de manejo en las evaluaciones e intervenciones psicológicas en el paciente de cuidados paliativos y su familia para evidenciar la contribución de la Psicología en Cuidados Paliativos.
\end{abstract}

Palabras clave: Psicólogos, Cuidados Paliativos, Análisis situacional

\section{[en] Situational analysis of palliative care psychologists}

\begin{abstract}
Introduction. Advanced chronic diseases patients have psychological, social and spiritual needs, as a result of their physical state, medical treatments, side effects, psychological and existential distress, so psychologists have great relevance in Palliative Care. Objective. Analyze the situation of the psychologists in the palliative care teams of public health units in Mexico. Method. Prospective, descriptive and cross-sectional study. An express survey of 22 items was designed with dichotomous response and multiple choice. Results. Analyzed 49 surveys of psychologists who provide services in medical care units in Mexico: analysis of frequencies, percentages, chi squared and ranks test' Wilcoxon. Conclusions. Palliative care psychologists require formal education and training to develop specific skills and competencies in this context; systematization and management protocols in evaluations and psychological interventions for patients and their families to demonstrate the contribution of psychologist in palliative care.
\end{abstract}

Keywords: Psychologists, palliative care, situational analysis.

1 Servicio de Cuidados Paliativos, Instituto Nacional de Cancerología. Avenida San Fernando 22, Belisario Domínguez Sección XVI, Tlalpan, Ciudad de México, 14080, México

E-mail: lascencioh@incan.edu.mx; leash71.1ah@gmail.com

* Dirección de correspondencia: Leticia Ascencio Huertas. Servicio de Cuidados Paliativos, Instituto Nacional de Cancerología. Avenida San Fernando 22, Belisario Domínguez Sección XVI, Tlalpan, Ciudad de México, 14080, México. E-mail: lascencioh@incan.edu.mx; leash71.lah@gmail.com 
Sumario. 1. Introducción 2. Método 3. Resultados 4. Discusión 5. Conclusión 6. Agradecimientos 7. Referencias bibliográficas

Cómo citar: Ascencio Huertas L. Análisis situacional de los psicologos de cuidados paliativos. Psicooncología 2020; 17: 357-373. doi: 10.5209/psic.71362

\section{Introducción}

Los pacientes con enfermedades crónicas en fase avanzada tienen numerosas necesidades psico-socio-espirituales como resultado de su estado físico, condición, curso terapéutico y efectos secundarios del tratamiento ${ }^{(1-3)}$ que impactan en su calidad de vida; ante lo cual los cuidados paliativos son una alternativa para coadyuvar al control de estas necesidades a través de un abordaje integral, holístico y centrado en la persona $a^{(4,5)}$.

Uno de los profesionales dentro del equipo de cuidados paliativos que puede ayudar con estas necesidades psico-socio-espirituales es el Psicólogo, a través de la detección y tratamiento de los trastornos emocionales ${ }^{(6)}$, la promoción de comportamientos de salud, la disminución de ingresos hospitalarios recurrentes y a los servicios de urgencias y los costos por dichos ingresos ${ }^{(7)}$.

Algunos estudios han demostrado que la mayoría de las interconsultas que se solicitan a los equipos de cuidados paliativos para pacientes ambulatorios no son solo por problemas relacionados con el control de síntomas físicos, sino también por el distrés o malestar psicológico como ansiedad, depresión y trastornos adaptativos, problemas psicológicos relacionados con las pérdidas actuales y anticipadas, el sufrimiento, el miedo a la muerte, la preocupación por sus seres queridos, el dolor total o descontrolado, el deseo de adelantar la muerte, el impacto emocional por la discapacidad, la disminución de la capacidad para la toma de decisiones al final de la vida y en el funcionamiento psicosocial de los cuidadores primarios y la familia ${ }^{(8-12)}$.

Sin embargo, a pesar de tener la certeza de que contar con la presencia del Psicólogo coadyuva al control de los síntomas y necesidades de pacientes en Cuidados Paliativos no existe una formación profesional formal hasta el momento en México para desarrollar las competencias específicas que se requieren.

A nivel internacional encontramos diversas propuestas, que difieren entre sí con respecto a las competencias necesarias con las que debe contar un Psicólogo en Cuidados Paliativos ${ }^{(13)}$. Una propuesta es la de Haley et al..$^{(14)}$ que han establecido que el psicólogo en este campo debe contar con conocimientos y habilidades para contribuir a la atención a pacientes y familias que enfrentan enfermedades potencialmente mortales y la muerte. Desde esta perspectiva, las habilidades del Psicólogo comprenden 3 niveles: (a) habilidades básicas para establecer y mantener relaciones de apoyo, (b) habilidades para emprender intervenciones psicológicas específicas para un problema / trastorno clínico, y (c) avanzadas para poder ayudar a pacientes con enfermedades crónicas en fase avanzada desde su diagnóstico, tratamiento, progresión, y durante el proceso de muerte, así como a los familiares en el duelo. Otros estudios mencionan que el psicólogo que brinde atención a pacientes con enfermedades crónicas en fase avanzada debe contar con supervisión clínica, apoyo personal, social y cultural, con una adecuada sensibilidad, ética, capacidad de 
autoconciencia y autorreflexión, que sea capaz de evaluar los efectos y calidad de las intervenciones psicológicas y con disposición para trabajar en equipo tanto con psicólogos clínicos como con otros profesionales de la salud ${ }^{(7,15,16)}$.

Por lo que es claro que el Psicólogo en Cuidados Paliativos debe contar con competencias cognitivas y habilidades clínicas para evaluar y atender la diversidad de psicopatologías e integrar los aspectos físicos sociales, culturales y espirituales y coadyuvar en los síntomas físicos.

Dentro de las principales funciones que debería realizar el psicólogo en las unidades de Cuidados Paliativos (CP), estás varían dependiendo de cada autor. Uno de ellos es el reportado por Jünger, Payne, Costantini, Kalus \& Werth ${ }^{(15)}$ y The National Institute for Health and Clinical Excellence Guidance (NICE) ${ }^{(17)}$, quienes reportan que el rol del psicólogo en las decisiones al final de la vida y la calidad del cuidado se basa en una estructura piramidal de cuatro niveles: los niveles I y II se caracterizan por el uso de técnicas generales de apoyo emocional donde participa el psicólogo y el resto del personal de salud de CP y los niveles III y IV, corresponden a las intervenciones específicas de counselling, manejo de la ansiedad, o técnicas más específicas (como la psicoterapia propiamente dicha) donde son competencias exclusivas del profesional de Psicología.

Por otra parte, The European Association for Palliative Care (EAPC), identificó 5 habilidades clave que los psicólogos aportan a un entorno de cuidados paliativos, que incluyen: 1) Evaluación psicológica, 2) Terapia psicológica para individuos, familias y grupos, 3) Consulta, supervisión y apoyo del personal, 4) Educación y 5) Investigación ${ }^{(15)}$.

Otro modelo es el propuesto por Payne y Haines ${ }^{(18)}$ que hace referencia a tres niveles de cuidado psicológico, cada uno de los cuales necesita de unas destrezas específicas para llevarse a cabo. El primero de ellos supone la utilización de habilidades básicas de comunicación y relación, el segundo requiere de una formación específica y el tercer nivel requiere de un amplio conocimiento teórico/ práctico de multitud de teorías, y se caracteriza por poder adaptar las habilidades a la problemática del paciente.

Y el modelo propuesto por Bayés ${ }^{(19,20)}$ que subraya la existencia de otros tres niveles: 1) detección del problema, 2) valoración de los síntomas psicológicos refractarios, tratamiento de síntomas como la angustia y del duelo y 3 ) intervenciones en el equipo de salud; para poder sistematizar, y evidenciar la efectividad de las intervenciones psicológicas en Cuidados Paliativos ${ }^{(21)}$.

Sin embargo, más allá de proporcionar atención clínica, los psicólogos en Cuidados Paliativos a menudo sirven como consultores para el equipo, programadores y evaluadores, líderes de equipo, profesores / supervisores e investigadores ${ }^{(22)}$.

También suelen gestionar pacientes complejos; como cuando niños o adolescentes están involucrados, ante dinámicas familiares que favorecen la conspiración de silencio, o en procesos de duelo complicado ${ }^{(23)}$, aplicar métodos de investigación para mejorar la calidad de la atención en apoyo a los objetivos de cuidados paliativos, desarrollar herramientas de evaluación de programas para determinar la eficacia de las intervenciones; e implementar y difundir modelos de atención ${ }^{(24,25)}$.

Sin embargo, aun cuando los psicólogos tienen mucho que ofrecer en el campo de los cuidados paliativos tanto en el ámbito clínico como en el de investigación, están subvalorados en las organizaciones profesionales de cuidados paliativos ${ }^{(26) \text {. }}$ Evidencia de ello es lo que se ha reportado Hoerger, Wayser, Schwing, Suzuki, 
Perry ${ }^{(27)}$ quienes reportaron que solo el $11 \%$ de los equipos de cuidados paliativos incluyen psicólogos y que la Psicología no ha desarrollado una especialidad de cuidados paliativos hasta ahora. Debido principalmente a que el psicólogo se encuentra cautivo de dos discursos diferentes: por un lado, en el que se habla de su importancia y que ve como un elemento básico y necesario en cualquier intervención, y otro se señala que sus funciones pueden ser realizadas por otros miembros del equipo ${ }^{(28)}$. Además de no contar una certificación profesional que regule la atención psicológica en estos escenarios, lo que limita las oportunidades para los psicólogos dentro y fuera de los hospitales ${ }^{(29)}$.

Por lo mencionado, para poder fortalecer a la Psicología dentro de los cuidados paliativos se realiza el presente estudio, con el objetivo de analizar el contexto actual (perfil profesional, procesos de atención clínica y determinantes laborales) de los psicólogos integrados a los equipos de cuidados paliativos del sector público en México.

\section{Método}

Estudio prospectivo, descriptivo y transversal en donde participaron psicólogos de unidades o servicios de cuidados paliativos en unidades de segundo y tercer nivel de atención en salud del sector público en México que respondieron a una convocatoria abierta a todas las unidades de Cuidados Paliativos de segundo y tercer nivel donde un profesional de Psicología formará parte del equipo. Una vez identificado al Psicólogo se le contacto por vía electrónica y se le invitó a participar voluntariamente en el estudio, una vez que aceptaba, se le hacía llegar por vía electrónica la encuesta creada para este estudio y una breve descripción del objetivo principal de la investigación. Se solicitó a los participantes que en un lapso de 15 días hábiles fuera reenviada la encuesta por vía correo electrónico a la investigadora. Se recibieron un total de 57 encuestas, de las cuales 49 cumplían con los criterios de inclusión (ser psicólogo de profesión, con título profesional, que brindara sus servicios a pacientes de cuidados paliativos), fueron eliminadas 8 encuestas de las cuales 5 laboraban en institución privada y que atendían pacientes de cuidados paliativos de forma eventual y 3 más que estaban incompletas.

\section{Instrumento}

La encuesta ex profeso fue desarrollada a partir de: a) revisión documental con respecto al rol de la Psicología en la atención de pacientes en cuidados paliativos; $b$ ) conformación un Comité de Psicólogos expertos en cuidados paliativos para establecer los bloques temáticos que engloban los principales problemas a lo que se enfrentan los psicólogos Cuidados Paliativos; c) creación de una matriz de priorización para identificar los problemas relacionados con los principales problemas que afrontan los psicólogos en Cuidados Paliativos a partir de 4 criterios: magnitud, frecuencia, gravedad y factibilidad. d) discernimiento de los expertos para establecer la prioridad los rubros a evaluar: 1) baja, 2) media y 3) alta. La versión final fue de 22 reactivos con tipo de respuesta dicotómica y opción múltiple. 


\section{Resultados}

La muestra total estuvo conformada por 49 encuestas, de las cuales $21(42,8 \%)$ fueron de instituciones de salud en el sector público de segundo nivel de atención (Hospitales Generales, Regionales, Integrales, Comunitarios; Hospitales Pediátricos, de Gineco-Obstetricia o Materno-Infantiles, así como los Hospitales Federales de Referencia) y $28(57,2 \%)$ y de tercer nivel (Centros Médicos Nacionales, Unidades Médicas de Alta Especialidad, los Institutos Nacionales de Salud).

Se realizaron análisis de frecuencias y porcentajes, prueba de normalidad shapiro-wilk (ya que la muestra es de $<50$ participantes en la que se comprobó que no es una distribución normal y que las muestras son bastante similares), pruebas no paramétricas: chi cuadrada para las variables categóricas y w de wilcoxon para las variables numéricas.

La Tabla 1 muestra que dentro de las características y procesos laborales de la atención psicológica de Cuidados Paliativos, se encontró que la Secretaría de Salud es el principal proveedor que cuenta con psicólogos en cuidados paliativos (segundo nivel $=95,2 \%$; tercer nivel $=60,7 \%$ ), en su mayoría concentrados en la Ciudad de México (segundo nivel=38,1\%; tercer nivel=46,4\%), se encuentran integrados principalmente en las Unidades o Servicios de Cuidados Paliativos de segundo nivel $(\mathrm{n}=9 / 42,9 \%)$ y Clínicas del dolor y Cuidados paliativos para el tercer nivel $(\mathrm{n}=8 / 28,6 \%)$; en equipos confirmados por un médico especialista, enfermera, psicólogo, trabajador social y nutriólogo (segundo nivel $n=11 / 52,4 \%$ y tercer nivel $\mathrm{n}=11 / 39,3 \% ; \mathrm{p}=0,225$ ), principalmente en el turno matutino (segundo nivel $n=19 / 90.5 \%$; tercer nivel $n=24 / 85,7 \% ; p=0,65)$.

Con respecto al estatus laboral se encontró que los psicólogos de tercer nivel son trabajadores de base, es decir, cuentan con seguridad laboral $(\mathrm{N}=20,71,4 \%)$ en comparación con el segundo nivel $(n=8 / 38,1 \%) ;(p=0,047)$, donde existe mayor inestabilidad. Más del $80 \%$ cuenta con un espacio físico (segundo nivel $n=19 / 90.5 \%$ y tercer nivel $n=24 / 85,7 \%$; $p=0,615$ ), sin embargo, es compartido con algún otro servicio o profesional de la salud (segundo nivel $n=15 / 71,4 \%$ y tercer nivel $\mathrm{n}=6 / 28,6 \% ; \mathrm{p}=0,305)$.

Con respecto a la jornada diaria de trabajo, tanto en el segundo y tercer nivel de atención es de 8 horas diarias $(\mathrm{p}=0,827)$, con relación a los años de experiencia clínica se encontró que en el segundo nivel se obtuvo una mediana de 10 años $(\mathrm{RIQ}=1-40)$ y para el tercer nivel una mediana de 11 años $(\mathrm{RIQ}=3-26)$, con una $\mathrm{p}=0.297$; en cuanto a la experiencia en la atención de pacientes terminales fue de 5 años $(\mathrm{RIQ}=1-11)$ para el segundo nivel y 7 años $(\mathrm{RIQ}=3-26)$ para tercer nivel $(\mathrm{p}=0,127)$; considerando el tiempo que lleva de haberse integrado formalmente el equipo de cuidados paliativos para el segundo nivel fue de 43 meses $(\mathrm{RIQ}=1-132)$ y para tercer nivel de 60 meses (RIQ=1-180); $(\mathrm{p}=0,669)$.

En el proceso de atención en sí, se encontró que el tiempo de consulta en pacientes de primera vez fue de 60 minutos $(\mathrm{RIQ}=30-120)$ para segundo nivel y de 50 minutos $(\mathrm{RIQ}=30-120)$ para tercer nivel $(\mathrm{p}=0,302)$; para consultas subsecuentes de 45 minutos $(\mathrm{RIQ}=30-60)$ para segundo nivel y 40 " $(\mathrm{RIQ}=20-60)$ para tercer nivel $(\mathrm{p}=0,554)$.

Dentro de las principales actividades que realizan los psicólogos en Cuidados paliativos prevalece la atención clínica tanto en el segundo nivel de atención $(\overline{\mathrm{x}}=85,71 ; 17,7 \%)$ como en tercer nivel de atención $(\overline{\mathrm{x}}=79,39 ; 17 \%)$ con una $\mathrm{p}=0,75$ por lo que no hay diferencias significativas. 


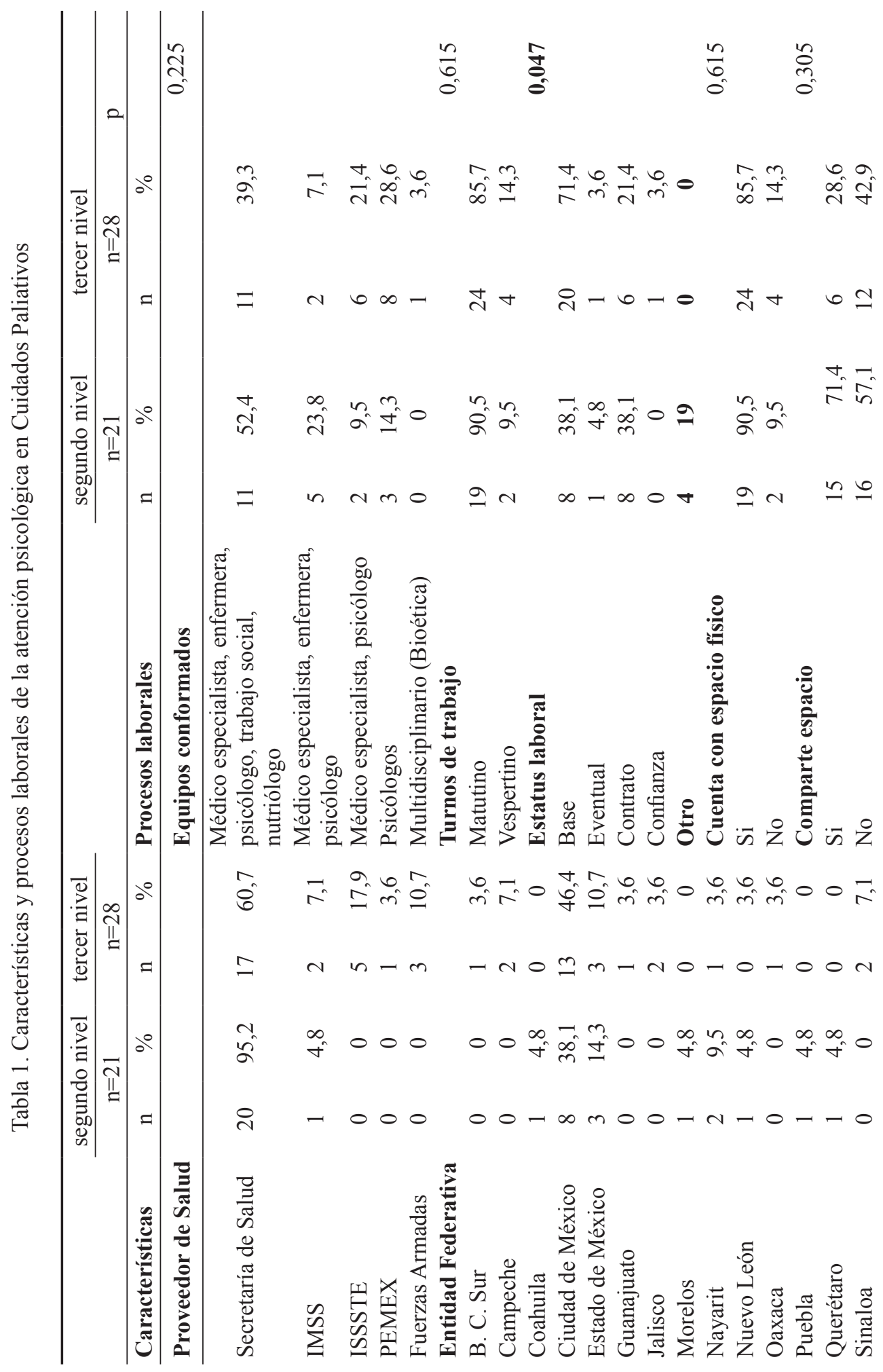




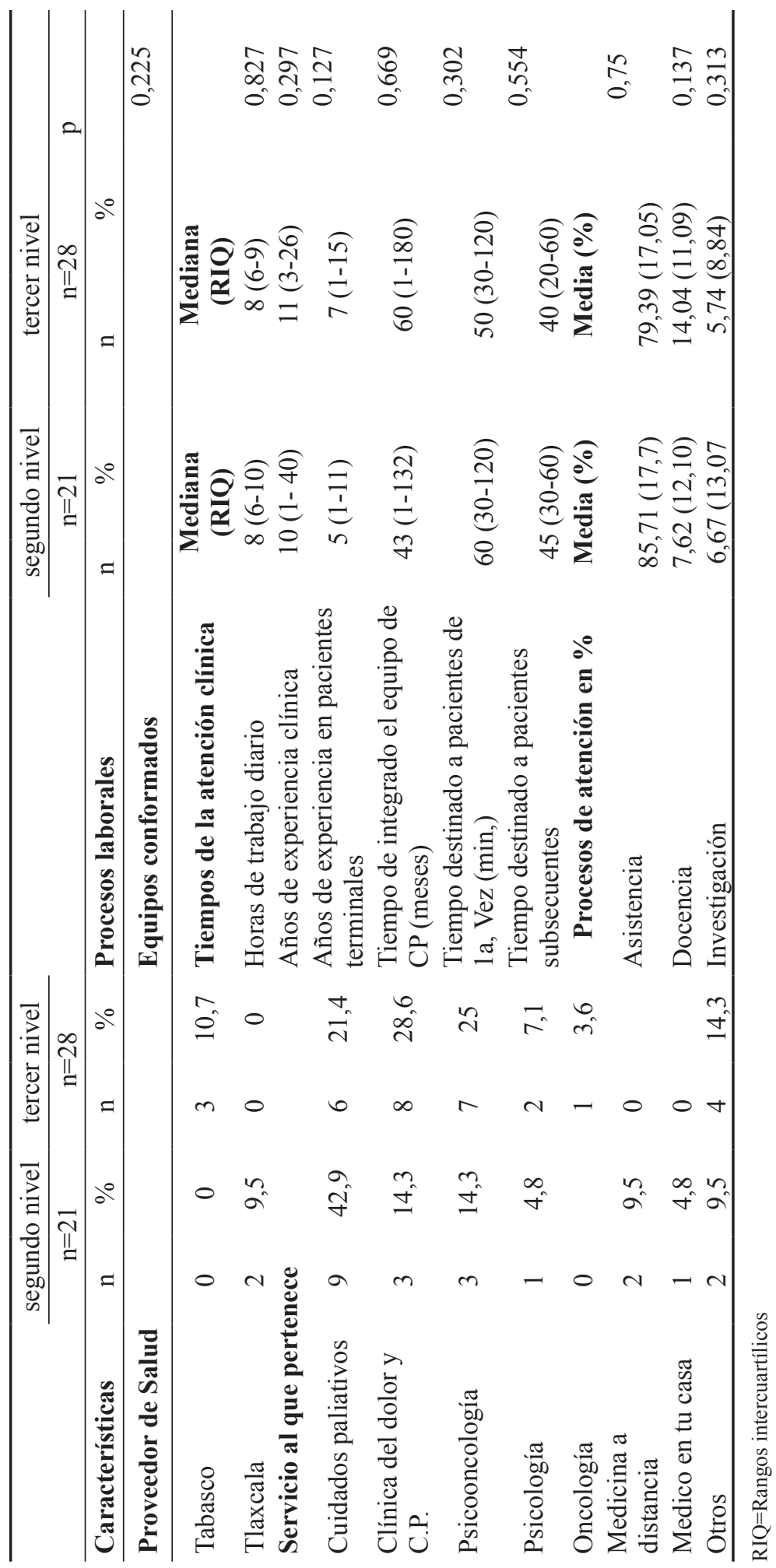


En cuanto a las actividades asistenciales, se encontró que la principal patología médica atendida en cuidados paliativos es el cáncer tanto para el segundo nivel (90,5\%) como para el tercer nivel $(96,4 \%)$, seguido de pacientes con diabetes mellitus (segundo nivel $=76,2 \%$ y tercer nivel $=60,7 \%$ ); en su mayoría adultos y geriátricos (segundo y tercer nivel en el 100\%) y en donde el principal tipo de intervención psicológica de tipo individual (segundo nivel $=85,7 \%$ y tercer nivel $=75 \%$ ); seguidas de las intervenciones de pareja y muy poco en intervenciones familiares y de grupo (Ver Figura 1).

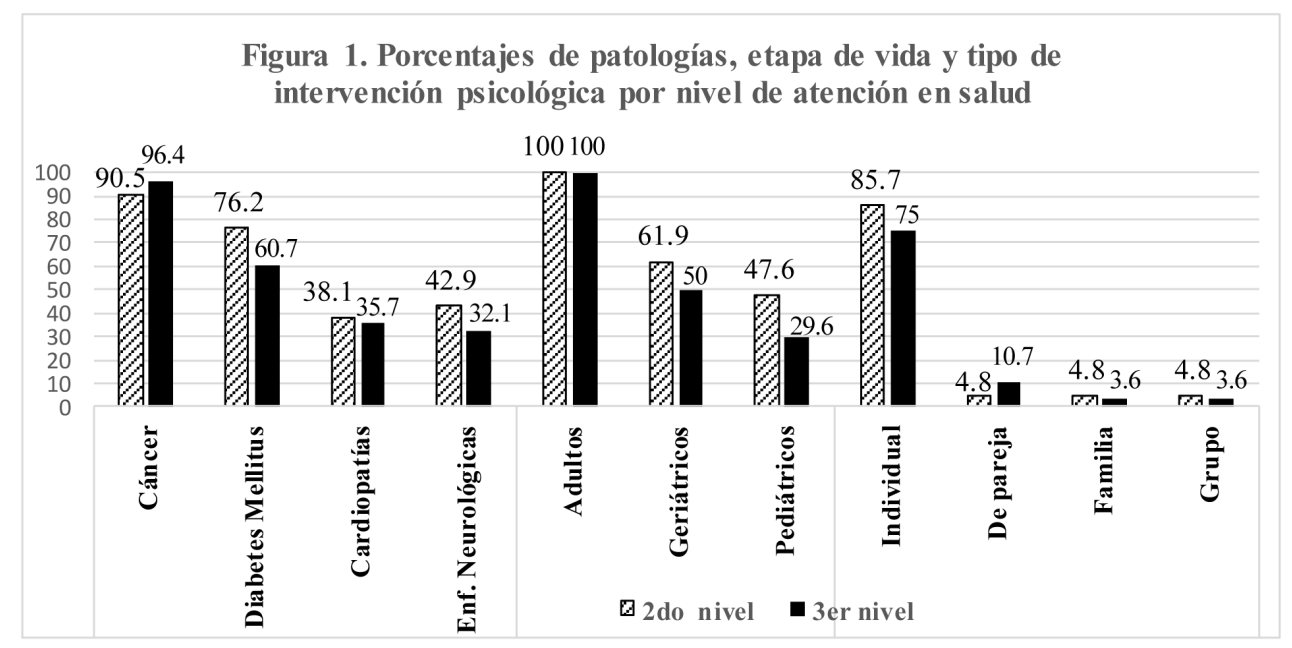

Lo relacionado con la formación académica, la Tabla 2 muestra que los psicólogos de segundo nivel cuentan en su mayoría con la licenciatura en Psicología $(\mathrm{n}=11 / 52,4 \%)$ y el tercer nivel cuentan con estudios de posgrado (maestría) $(\mathrm{n}=12 / 42,9 \%) \mathrm{p}=0,154$; la mitad de ellos ha realizado Diplomados en Cuidados Paliativos y/o Tanatología (segundo nivel: $n=11 / 52,4 \%$; tercer nivel: $n=13 / 46,4 \%$, con una $\mathrm{p}=0,279$ ); predominando los de tipo presencial (segundo nivel $\mathrm{n}=17 / 81 \%$, tercer nivel $n=18 / 64,3 \% ; p=0,186)$. Y el Modelo teórico que prevalece es el CognitivoConductual (TCC) en ambos niveles de atención (segundo nivel $n=12 / 57,1$; tercer nivel $n=22 / 78,6 ; p=0,186)$.

Tabla 2. Formación académica de los psicólogos en Cuidados Paliativos

\begin{tabular}{lccccc}
\hline & \multicolumn{2}{c}{ segundo nivel } & \multicolumn{2}{c}{ tercer nivel } \\
$\mathrm{n}=21$ & $\mathrm{n}=28$ & $\mathrm{p}$ \\
Características académicas & $\mathrm{N}$ & $\%$ & $\mathrm{n}$ & $\%$ & \\
\hline Formación profesional & & & & & 0,154 \\
Licenciatura & 11 & 52,4 & 7 & 25 & \\
Maestría & 8 & 38,1 & 12 & 42,9 & \\
Especialidad & 1 & 4,8 & 4 & 14,3 & \\
Doctorado & 1 & 4,8 & 5 & 17,9 & \\
\hline
\end{tabular}




\begin{tabular}{|c|c|c|c|c|c|}
\hline \multirow[b]{2}{*}{ Características académicas } & \multicolumn{2}{|c|}{$\begin{array}{c}\text { segundo nivel } \\
n=21\end{array}$} & \multicolumn{2}{|c|}{$\begin{array}{c}\text { tercer nivel } \\
n=28\end{array}$} & \multirow[t]{2}{*}{$\mathrm{p}$} \\
\hline & $\mathrm{N}$ & $\%$ & $\mathrm{n}$ & $\%$ & \\
\hline \multicolumn{3}{|c|}{ Formación en cuidados paliativos } & & & 0,279 \\
\hline Curso & 10 & 47,7 & 9 & 32,1 & \\
\hline Diplomado & 11 & 52,4 & 13 & 46,4 & \\
\hline Maestría & 0 & 0 & 1 & 3,6 & \\
\hline Máster & 0 & 0 & 1 & 3,6 & \\
\hline Sin formación formal en $\mathrm{CP}$ & 0 & 0 & 4 & 14,3 & \\
\hline Modalidad & & & & & 0,498 \\
\hline Presencial & 17 & 81 & 18 & 64,3 & \\
\hline En línea & 3 & 14,3 & 5 & 17,9 & \\
\hline Mixto & 0 & & 1 & 3,6 & \\
\hline Autodidacta & 1 & 4,8 & 4 & 14,3 & \\
\hline Marco teórico & & & & & 0,186 \\
\hline Cognitivo-conductual & 12 & 57,1 & 22 & 78,6 & \\
\hline Psicoanálisis & 2 & 9,5 & 3 & 10,7 & \\
\hline Humanista & 2 & 9,5 & 3 & 10,7 & \\
\hline Gestalt & 2 & 9,5 & 0 & 0 & \\
\hline Sistémico & 1 & 4,8 & 0 & 0 & \\
\hline Otro & 2 & 9,5 & 0 & 0 & \\
\hline
\end{tabular}

$\mathrm{p}<0,05$

Con respecto a la evaluación psicológica se encontró que tanto en el segundo $(n=12 / 57,1 \%)$ como en el tercer nivel $(n=18 / 64,3 \%)$ se utiliza al menos un instrumento de evaluación para la detección de necesidades de atención, sin diferencias significativas $(\mathrm{p}=0,415)$. Las principales áreas por evaluar con algunos instrumentos ya validados y estandarizados en México que se encontraron: Estado cognitivo, depresión y ansiedad, detección de necesidades, apoyo social y familiar, calidad de vida, personalidad, cuidador primario informal, se enlistan en la Tabla 3.

Tabla 3. Escalas de evaluación más utilizadas por los psicólogos en Cuidados Paliativos en segundo y tercer nivel de atención en México.

\section{Escalas del estado cognitivo}

Short Portable Mental Status Questionnaire de Pfeiffer (SPMSQ)
Martínez, Dueñas, Onís, Aguado, Albert y Luque, 2001(30).

\section{Escalas de depresión y ansiedad}

Cuestionario para simplificar el diagnóstico del Síndrome depresivo
Calderón, 1992(31); Morales, Ocampo, y Alvarado, 1996 ${ }^{(32)}$. 
Inventario de depresión de Beck

Inventario de ansiedad de Beck

Escala de depresión, ansiedad y estrés (DASS-21)

Escala Hospitalaria de ansiedad y depresión (HADS)

Escala de depresión de posparto de Edimburgo

Inventario de ansiedad rasgo-estado (IDARE)
Jurado, Villegas, Méndez, Rodríguez, Loperena y Varela, $1998^{(33)}$.

Robles, Varela, Jurado y Páez, $2001^{(34)}$.

Daza., Novy, Stanley y Averill, $2002^{(35)}$

Galindo, Benitet, Juárez, Rojas, Riveros, Aguilar, et. al. 2015 ${ }^{(36)}$.

Alvarado, Sifuentes, Salas y Martínez, 2006 ${ }^{(37)}$.

Spielberger. Díaz Guerrero, $2002^{(38)}$. Guillén Díaz-Barriga y

González-Celis, 2009 ${ }^{(39)}$.

\section{Escalas de detección de necesidades}

Entrevista para la detección de necesidades psicosociales en enfermedad avanzada (DENEPSI)

Termómetro de distrés

Evaluación

de las Necesidades de Pacientes Oncológicos

en Etapa Terminal (ENPOET)
Rivera, Mejía, Chiquete, Beltrán, Barrientos, Alfaro et al., $2016^{(40)}$.

Almanza, Juárez \& Pérez, 2008(41).

Reynoso, Alazraki, GonzálezMaraña, Alvarado y Pulidol, $2008^{(42)}$.

\section{Escalas de apoyo familiar y social}

Apgar familiar

Escala de apoyo social (MOS)
Gómez y Ponce, 2010 ${ }^{(43)}$.

Martínez et al., 2014(44).

\section{Escalas de Calidad de Vida}

Inventario de Calidad de vida y salud (INCAVISA) Riveros, Sánchez-Sosa \& Del Aguila, 2008 ${ }^{(45)}$.

\section{Escalas de Personalidad}

Inventario Multifásico de la personalidad (MMPI-2) Butcher, Graham, Ben-Porath, Tellegen et al., 2015 $5^{(46)}$.

Test de frases incompletas de Sacks Galvis y Montemayor, $1971^{(47)}$.

\section{Escalas para el Cuidador primario informal}

Escala de sobrecarga del cuidador primario de Zarit

Pardo, Cardenas, Cruz, Venegas y Magaña, 2014(48).

\section{Discusión}

Dentro de las conclusiones más importante encontramos que los psicólogos en cuidados paliativos se encuentran en el segundo y tercer niveles de atención y concentrados en las grandes ciudades en México, no encontrando hasta ahora un registro de los psicólogos insertados en el ler nivel de atención donde también se tiene una gran necesidad de apoyo psicológico para la atención de pacientes de cuidados paliativos en domicilio y la comunidad. 
De acuerdo con los datos obtenidos, no es suficiente el número de profesionales de la Psicología insertados en Cuidados Paliativos ya que se requiere de un mayor número de estos, para cubrir todas las necesidades que giran alrededor de la atención de la población que requerirá en un futuro de Cuidados paliativos, principalmente por el crecimiento de las enfermedades crónico-degenerativas o enfermedades crónicas no transmisibles, tanto en hombres como en mujeres, en México y en el mundo ${ }^{(49-51)}$.

Sin embargo vale la pena relatar que los psicólogos que brindan atención psicosocio-espiritual tanto en el segundo como en el tercer nivel formen parte de equipos completos de profesionales de la salud lo cual promueve un abordaje holístico y transdisciplinario; no obstante que la mayoría de ellos presta sus servicios solo en el turno matutino, dejando descubierto tanto el turno vespertino, nocturno e incluso fines de semana.

Es evidente al analizar las condiciones de trabajo, la necesidad de contar con mayor seguridad laboral en los psicólogos, ya que son muy pocos los que cuentan con ella, principalmente los de tercer nivel de atención (Hospitales de Alta Especialidad o Institutos Nacionales de Salud).

Con respecto a los espacios físicos es necesario promover espacios físicos adecuados y suficientes para llevar a cabo intervenciones psicológicas que permita cumplir con 2 principios éticos básicos en psicoterapia: confidencialidad y privacidad, además de que se menciona en la normativa establecida en cuidados paliativos, donde se establece la necesidad de contar con un consultorio destinado a la atención psicológica y una sala de familiares para actividades con la familia y abordajes grupales ${ }^{(52,53)}$.

La jornada laboral diaria que es de 8 horas en promedio, cuyo tiempo destinado para cada paciente de primera vez va desde 50 a 60 minutos y para los subsecuentes es de 40 a 45 minutos; lo que impacta en la productividad diaria de los psicólogos en cuidados paliativos, y que, al ser comparado con la cantidad de pacientes atendidos por el área médica en instituciones públicas, existe una franca desventaja como lo establece Julio Alfonso Piña ${ }^{(54)}$.

Sin embargo, es importante mencionar que la decisión acerca de cuánto tiempo el profesional de psicología estará cara a cara con el paciente, resulta vital no sólo para programar la agenda de turnos sino también para garantizar la calidad de atención. Ya que una consulta demasiado corta ha sido descrita, entre otras cosas, como uno de los factores que impide la realización de una buena intervención psicológica, aun cuando no se ha reportado una duración óptima para cumplir con los objetivos terapéuticos como lo establece Outomuro y Actis ${ }^{(55)}$.

Uno de los resultados que llama la atención es que el personal de Psicología cuenta con experiencia tanto clínica como en la atención de pacientes terminales aún mayor al tiempo que fueron conformados los equipos de cuidados paliativos, lo cual indica que ya se brindaba atención psicológica a pacientes con enfermedades crónicas avanzadas como interconsultantes, pero no como parte del equipo multi y transdisciplinario de Cuidados Paliativos.

Con respecto a la formación académica es evidente que los psicólogos de CP cuentan con mayor grado académico en el tercer nivel de atención en salud, sin embargo no son posgrados orientados a Cuidados Paliativos sino que están basados en Psicología Clínica y de Salud y lo han complementado con cursos o diplomados en Cuidados Paliativos y Tanatología, lo que hace evidente la necesidad de contar con una formación profesionalizante a través de un posgrado o capacitación orientada 
en Cuidados paliativos para fortalecer y desarrollar las competencias profesionales de los psicólogos que están o serán insertados en las Unidades de Cuidados Paliativos en cualesquiera de los niveles de atención. Al respecto, Payne \& Haines ${ }^{(18)}$ mencionan que en Gran Bretaña los psicólogos clínicos deben complementan su formación académica y profesional con 3 años más como requisito para el desarrollo de habilidades clínicas y conocimientos a través de la exposición a una amplia gama de enfoques terapéuticos y competencias en la evaluación, formulación clínica e intervención de problemas psicológicos experimentados por personas con enfermedades avanzadas y al final de la vida.

Al respecto son varios estudios han reportado la necesidad de contar con una profesionalización de los psicólogos en el campo de los Cuidados Paliativos ${ }^{(56)}$. En Europa, el informe final de la Federación Europea de Asociaciones de Psicólogos Profesionales (EFPA) especificó que los psicólogos tienen la responsabilidad de capacitarse y tener la experiencia adecuada para garantizar que solo practican en áreas de su competencia y señaló los componentes necesarios de la capacitación: (1) base de conocimiento académico (psicología); (2) base de conocimiento académico, (otro) (3) aplicación de habilidades psicológicas para el cuidado de la salud; (4) habilidades de investigación; (5) habilidades de enseñanza y capacitación; (6) habilidades de gestión; (7) cuestiones profesionales; y (8) cuestiones éticas ${ }^{(57)}$. Por lo que se es urgente contar con estudios de posgrado en Psicología en materia de cuidados paliativos ${ }^{(58)}$ incluido México, donde hasta el momento no se cuenta con una formación profesional de posgrado o capacitación formal para los psicólogos en el contexto paliativo.

Uno de los enfoques psicológicos que ha resultado ser más eficaz para la atención de pacientes de Cuidados Paliativos es el cognitivo conductual, probablemente por que se centra en el aquí y ahora, además de ser simple y breve, y contar con esta capacitación en TCC se pueden fortalecer las habilidades y competencias específicas para el contexto de la salud ${ }^{(59)}$.

Uno de los dolores de cabeza de los psicólogos es contar con herramientas o instrumentos que permitan evidenciar la eficacia de las intervenciones. Por lo que una e las propuestas a raíz de este trabajo es unificar tanto como sea posible el uso de Escalas básicas para la detección de necesidades psico-socio-espirituales y un método de evaluación que nos permita valorar la efectividad y tamaño del efecto de las intervenciones psicológicas ${ }^{(60)}$. Ya que son pocos los que llevan a cabo estos procesos de forma estandarizada, situación que coincide con lo reportado por Nakajima y cols. (56) al mencionar que solo el $50 \%$ de los psicólogos realiza evaluaciones del estado mental de pacientes, familiares y profesionales de salud.

Dentro de las áreas de oportunidad que se propone subrayar es que dentro del abordaje psicológicos se incluya la evaluación y manejo de los aspectos espirituales, el manejo de grupos, la intervención con niños y adolescentes, no solo para los cuidados paliativos pediátricos sino como familiares de pacientes que pueden ser sus padres, abuelos o figuras significativas y con un lazo afectivo importante.

Otro punto de pendiente es el de impulsar que dentro de las principales funciones del psicólogo en las unidades de cuidados paliativos no solo deberían ser en la atención clínica sino también actividades docentes, de investigación, incluso de biopolítica como lo establece Fernández et al. ${ }^{(28)}$.

Dentro de las limitaciones en el presente estudio, se encuentra la metodología utilizada para la elaboración de la encuesta, ya que, al realizar un trabajo en equipo 
con otros especialistas o expertos en el campo, puede contar con una herramienta de evaluación más completa, por lo que para futuras investigación en el campo se sugiere diseñar un cuestionario o encuesta con mayores fortalezas metodológicas. Y considerar la participación de los psicólogos en cuidados paliativos pediátricos y en primer nivel atención en salud.

\section{Conclusiones}

Basado en el análisis funcional podemos concluir que aun cuando la Psicología se ha insertado en los contextos hospitalarios, todavía existen varios obstáculos para que los psicólogos sean considerados como profesionales indispensables en la atención integral y holística en Cuidados paliativos. Uno de ellos es el desconocimiento de aquellas funciones y condiciones en las que el psicólogo en cuidados paliativos debe incidir y la pobre evidencia científica acerca de su actuar profesional, ya que la mayoría se ha enfocado a la atención clínica y ha descuidado la docencia y la investigación, que afecta directamente la producción de conocimiento y evidencia que puede apoyar tales prácticas.

Por lo que es necesario fortalecer la formación y capacitación formal de los psicólogos en Cuidados Paliativos para poder impactar en los 3 niveles de atención en salud en México y sistematizar los procesos de evaluación psico-socio-espiritual y poder medir la eficacia y efectividad de las intervenciones para elucidar el papel del psicólogo y contribuir efectivamente al propósito de los cuidados paliativos.

\section{Referencias bibliográficas}

1. Iwamitsu Y, Hirai K, Oba A, Shiozaki M, Asai M, Ogata A, et al. Roles of clinical psychologists desired by palliative care team members: Focus group interviews. Palliat Care Res 2009;2:228-34. doi:10.2512/jspm.4.228

2. Mitchell AJ, Chan M, Bhatti H, Halton M, Grassi L, Johansen C, et al. Prevalence of depression, anxiety, and adjustment disorder in oncological, haematological, and palliative-care settings: a meta-analysis of 94 interview-based studies. Lancet Oncol 2011;2:160-74. doi: 10.1016/S1470-2045(11)70002-X

3. Götze H, Brähler E, Gansera L, Polze N, Köhler N. Psychological distress and quality of life of palliative cancer patients and their caring relatives during home care. Support Care Cancer 2014;10:2775-82. doi:10.1007/s00520-014-2257-5

4. Boston P, Bruce A, Schreiber R. Existential suffering in the palliative care setting: An integrated literature review. J Pain Symptom Manage 2011; 3: 604-18. doi: 10.1016/j. jpainsymman.2010.05.010

5. Penner JL, Stevenson M, Parmar MP, Bélanger E. The psychosocial needs of students conducting research with patients and their families in advanced cancer and palliative care: A scoping review. Palliat Support Care 2017;2:260-6. doi: 10.1017/S1478951516000481.

6. Bakitas M, Lyons KD, Hegel MT, Balan S, Brokaw FC, Seville J, et al. Effects of a palliative care intervention on clinical outcomes in patients with advanced cancer: the project ENABLE II randomized controlled trial. JAMA 2009;7:741-9. doi: 10.1001/ jama.2009.1198 
7. Fan S-Y, Lin W-C, Lin I-M. Psychosocial care and the role of clinical psychologists in palliative care: Am J Hosp Palliat Med 2015;8:861-868. doi: 10.1177/1049909114543492

8. Breitbart W, Rosenfeld B, Pessin H, Kaim M, Funesti-Esch J, Galietta M, et al. Depression, hopelessness, and desire for hastened death in terminally ill patients with cancer. JAMA 2000; 22: 2907-11. doi:10.1001/jama.284.22.2907

9. Portenoy RK, Thaler HT, Kornblith AB, Lepore JM, Friedlander-Klar H, Coyle N, et al. Symptom prevalence, characteristics and distress in a cancer population. Qual Life Res Int J Qual Life Asp Treat Care Rehabil 1994;3:183-9. doi: 10.1007/BF00435383

10. Hermes HR, Lamarca ICA. Palliative care: an approach based on the professional health categories. Ciênc Amp Saúde Coletiva. 2013;9:2577-88. doi: 10.1590/S141381232013000900012

11. Azuero C, Allen RS, Kvale E, Azuero A, Parmelee P. Determinants of psychology service utilization in a palliative care outpatient population. Psychooncology 2014;6:650-7. doi: 10.1002/pon.3454.

12. Noreika DM, Coyne PJ. Implementing Palliative Care Interdisciplinary Teams: Consultative Versus Integrative Models. Crit Care Nurs Clin North Am 2015; 3: 297-306. doi: 10.1016/j.cnc.2015.05.006. doi10.1016/j.cnc.2015.05.006

13. Fernández MEF, Clemente PA. Estándares de formación psicológica en Cuidados Paliativos. Med Paliativa 2004;3:174.

14. Haley WE, Larson DG, Kasl-godley J, Neimeyer RA, Kwilosz DM. Roles for psychologists in end-of-life care: Emerging models of practice. Prof Psychol Res Pr 2003; 6: 626-33. doi: 10.1037/0735-7028.34.6.626

15. Jünger S, Payne S, Costantini A, Kalus C, Werth J. The EAPC TASK Force on education for psychologists in palliative care. Eur J Palliat Care. 2010;17:84-87. [Acceso 1 de julio de 2020]. Disponible en: https://eprints.lancs.ac.uk/id/eprint/32728/1/ JournalArchiveArticle.pdf

16. Jünger S, Payne S. Guidance on postgraduate education for psychologists involved in palliative care. Eur J Palliat Care 2011;5:238-52. [Acceso 1 de julio de 2020]- Disponible en: http://www.eapcnet.eu/LinkClick.aspx?fileticket=7kOIkiphMt0\%3D\&tabid=194

17. National Institute for Clinical Excellence. Guidance on cancer services: improving supportive and palliative care for adults with adults the manual. London: National Institute for Clinical Excellence; 2004.

18. Payne S, Haines R. The contribution of psychologists to specialist palliative care. Int J Palliat Nurs 2002;8:401-6. doi:10.12968/ijpn.2002.8.8.10684

19. Barreto MP. El psicólogo ante el enfermo en situación terminal. An Psicol 1990;6:169-80. [Acceso 1 de julio de 2020]. Disponible en: http://hdl.handle.net/10201/5311

20. Bayés R. Medicina Paliativa: psicología y Cuidados Paliativos. Med Paliativa 2005;3:137.

21. Schneider Donelli TM, de Castro EK. A Discussion about Health Psychology and Palliative Care in Brazil. Hosp Palliat Med Int J 2017;5:00028-00029. [Acceso 1 de julio de 2020]. Disponible en: https://medcraveonline.com/HPMIJ/a-discussion-about-healthpsychology-and-palliative-care-in-brazil.html

22. Nash JM, Khatri P, Cubic BA, Baird MA. Essential competencies for psychologists in patient centered medical homes. Prof Psychol Res Pract 2013;44:331-42. doi: 10.1037/ a0033106

23. Duro Martínez JC, De Miguel C, Morillo E, Noguera A, Núñez Portela B, Ruiz López D. Discurso de los profesionales de cuidados paliativos de la Comunidad de Madrid sobre la atención psicológica. Psicooncología 2013;9:467-81. doi: 10.5209/rev_PSIC.2013. v9.n2-3.40918 
24. Kazak AE, Nash JM, Hiroto K, Kaslow NJ. Psychologists in patient-centered medical homes (PCMHs): Roles, evidence, opportunities, and challenges. Am Psychol 2017;1:112. doi: $10.1037 / \mathrm{a} 0040382$

25. Ryan K, Connolly M, Charnley K, Ainscough A, Crinion J, Hayden C, et. al. Palliative Care Competence Framework. Dublin: Health Service Executive, 2014. [Acceso 1 de julio de 2020] Disponible de: https:/www.hse.ie/eng/services/publications/clinicalstrategy-and-programmes/palliative-care-framework-document.pdf

26. Hoerger M, Wayser GR, Schwing G, Suzuki A, Perry LM. Impact of interdisciplinary outpatient specialty palliative care on survival and quality of life in adults with advanced cancer: A meta-analysis of randomized controlled trials. Ann Behav Med 2019;7:674-85. doi: 10.1093/abm/kay077

27. Hoerger M, Ramos K, Ellington L, Perry LM, Pollak KI \& Porter LS. (2019). Organizing Psychologists, behavioral scientists, and allied professionals: formation of the Society of Behavioral Medicine's Palliative Care Special Interest Group. J Pain Symptom Manage 2019; 4: e10-e11. doi: 10.1016/j.jpainsymman.2019.06.002

28. Fernández-Alcántara M, Ortega-Valdivieso A, Pérez-Marfil MN, García-Caro MP, CruzQuintana F. Funciones y situación actual de la intervención de los psicólogos en cuidados paliativos. Psicooncología. 2014; 1: 163-72. doi: 10.5209/rev_PSIC. 2014.v11.n1.44925

29. Kodama K, Uchino T, Isobe N. A survey of activities of clinical psychologists in medical fields with highly advanced technology, Bull Train Res Cent Clin Psychol 2004; 3:185-91 (in Japanese).

30. Martínez-De la Iglesia J, Dueñas-Herrero R, Onís-Vilches MC, Aguado-Taberné C, AlbertColomer C, Luque-Luque R. Adaptación y validación al castellano del cuestionario de Pfeiffer (SPMSQ) para detectar la existencia del deterioro cognitivo en personas mayores de 65 años. Med Clin. 2001;4:129-34

31. Calderón NG. Cuestionario clínico para el diagnóstico de los cuadros depresivos. Rev Med IMSS (México) 1992; 30: 377-80.

32. Morales RM, Ocampo AVM, Alvarado CR. Validez y confiabilidad del cuestionario clínico de síndrome depresivo. Arch Neurocien 1996; 1: 11-15

33. Jurado S, Villegas ME, Méndez L, Rodríguez F, Loperena V y Varela R. La estandarización del Inventario de Depresión de Beck para los residentes de la ciudad de México. Salud Mental; 3:26-31.

34. Robles R, Varela R, Jurado S y Páez F. Versión mexicana del inventario de ansiedad de Beck: propiedades psicométricas. Revista Mexicana de Psicología.2001; 2:211-218.

35. Daza P, Novy DM, Stanley MA, y Averill P. The depression anxiety stress scale-21: Spanish translation and validation with a Hispanic sample. J Psychopathol Behav Assess 2002; 3: 195-205.

36. Galindo O, Benjet C, Juárez F, Rojas E, Riveros A, Aguilar JL, Alvarez MA, Alvarado S. Propiedades psicométricas de la Escala Hospitalaria de Ansiedad y Depresión (HADS) en una población de pacientes oncológicos mexicanos Salud Mental 2015; 4:253-8 doi: 10.17711/SM.0185-3325.2015.035

37. Alvarado-Esquivel C, Sifuentes-Alvarez A, Salas-Martinez C, Martínez-García S. Validation of the Edinburgh Postpartum Depression Scale in a population of puerperal women in Mexico. Clin Pract Epidemiol Ment Health 2006; 2:1-5 doi:10.1186/17450179-2-33

38. Spielberger. Ch., Díaz Guerrero R Inventario de Ansiedad Rasgo-Estado IDARE estandarizada en México. 2002 México: Manual Moderno ISBN: 28-100 
39. Guillén Díaz-Barriga, Centli, and Ana Luisa González-Celis Rangel. Propiedades psicométricas del Inventario de Ansiedad de Beck en adultos asmáticos mexicanos. Psicol Salud 2019; 1: 5-16 [Acceso 1 de julio de 2020]. Disponible en: https://psicologiaysalud. uv.mx/index.php/psicysalud/article/view/2563/4471

40. Rivera G, Mejía J, Chiquete, E, Beltrán C, Barrientos V. Alfaro I., et al. Evaluación de variables psicosociales en el cuidador del paciente con enfermedad avanzada: una propuesta preliminar. Psicol Salud, 2: 147-60. [Acceso 1 de julio de 2020]. Disponible en: https://psicologiaysalud.uv.mx/index.php/psicysalud/article/viewFile/2191/391

41. Almanza J.J., Juárez I.R., \& Pérez S. Traducción, adaptación y validación del termómetro de distrés en una muestra de paciente mexicanos con cáncer. Rev. Sanid Milit Mex 2008; 5: 209-17. [Acceso 1 de julio de 2020]. Disponible en: https://www.medigraphic.com/ pdfs/sanmil/sm-2008/sm085a.pdf

42. Reynoso E, Alazraki O, González-Maraña MC, Alvarado S, Pulidol MA. Evaluación de las necesidades de pacientes oncológicos en etapa terminal (a través del instrumento EMPOET): comparación entre muestras. Psicooncología 2008; 1: 39-52.

43. Gómez FJ, Ponce ER. Una nueva propuesta para la interpretación de Family APGAR (versión en español). Atención familiar 2010; 4: 102-6. doi: 10.22201/ facmed.14058871p.2010.4.21348

44. Martínez A, Sánchez S, Aguilar EJ, Rodriguez V y Riveros A. Adaptación y Validación del Cuestionario MOS de Apoyo Social en pacientes mexicanos con VIH+. Revista Latinoamericana de Medicina Conductual 2014; 2: 93-101 [Acceso 1 de julio de 2020]. Disponible en: https://www.redalyc.org/pdf/2830/283043156006.pdf

45. Riveros, A, Sánchez-Sosa, y Del Aguila M. Inventario de calidad de vida y salud. InCaVisa: 2008, México: Manual Moderno

46. Butcher, J., Graham, J., Ben-Porath, Y, Tellegen A., Dahlstrom W.G., Kaemmer B. Inventario Multifásico de la Personalidad Minnesota ${ }^{\circledR}-2$. Edición revisada MMPI-2 México: Manual Moderno

47. Galvis LM, Montemayor F. Estudio descriptivo del cuestionario proyectivo de Sacks. (Frases incompletas)." Anales del Museo Nacional de México. No. 2. Museo Nacional de México, 1971. [Acceso 1 de julio de 2020]. Disponible en: https://mediateca.inah.gob. $\mathrm{mx} /$ repositorio/islandora/object/articulo:7543

48. Montero Pardo X, Jurado Cardenas S, Valencia Cruz A, Méndez Venaga J, Mora Magaña I. Escala de carga del cuidador de Zarit: evidencia de validez en México. Psicooncología 2014; 1: 71-85. doi: 10.5209/rev_PSIC.2014.v11.n1.44918

49. INFORME_LA_SALUD_DE_LOS_MEXICANOS_2015_S.pdf[Internet]. [citado $27 \mathrm{de}$ julio de 2020]. Disponible en: https://www.gob.mx/cms/uploads/attachment/file/64176/ INFORME_LA_SALUD_DE_LOS_MEXICANOS_2015_S.pdf

50. ESMO. World Cancer Report 2014 [Internet]. [Acceso 28 de julio de 2020]. Disponible en: https://www.esmo.org/oncology-news/world-cancer-report-2014

51. Sathitratanacheewin S, Engelberg RA, Downey L, Lee RY, Fausto JA, Starks H, et al. Temporal trends between 2010 and 2015 in intensity of care at end-of-life for patients with chronic illness: Influence of age under vs. over 65 years. J Pain Symptom Manage 2018; 55: 75-81. doi: 10.1016/j.jpainsymman.2017.08.032

52. Decreto por el que se reforma y adiciona la Ley General de Salud en Materia de Cuidados Paliativos. DOF: 05/01/2009. [Acceso 1 de julio de 2020]. Disponible en: http://dof.gob. $\mathrm{mx} /$ nota_detalle.php? codigo $=5076793 \&$ fecha $=05 / 01 / 2009$

53. Norma Oficial Mexicana NOM-011-SSA3-2014, Criterios para la atención de enfermos en situación terminal a través de cuidados paliativos. DOF: 09/12/2014. [Acceso 1 de 
julio de 2020]. Disponible en: http://www.dof.gob.mx/nota_detalle.php?codigo=537501 9\&fecha $=09 / 12 / 2014$

54. Piña-López JA. La Psicología y los psicólogos en el sector de la salud en México: algunas realidades perturbadoras. 2004; 1: 191-205. [Acceso 1 de julio de 2020]. Disponible en: https://www.redalyc.org/articulo.oa?id=33740111

55. Outomuro D, Actis AM. Estimación del tiempo de consulta ambulatoria en clínica médica. Rev Méd Chile 2013;3: 361-6. doi: 10.4067/S0034-98872013000300012

56. Nakajima K, Iwamitsu Y, Matsubara M, Oba A, Hirai K, Morita T, et al. Psychologists involved in cancer palliative care in Japan: A nationwide survey. Palliat Support Care 2015; 2: 327-34. doi:10.1017/S1478951514000029

57. Marks DF, Brücher-Albers C, Donker FJS, Jepsen Z, Rodriguez-Marin J, Sidot S et al. Health Psychology 2000:The Development of Professional Health Psychology: European Federation of Professional Psychologists' Associations (EFPPA) Task Force on Health Psychology Final Report. J Health Psychol 1998; 3: 149-60. doi:10.1177/135910539800300112

58. Iwamitsu Y, Oba A, Hirai K, Asai M, Murakami N, Matsubara M, et al. Troubles and hardships faced by psychologists in cancer care. Jpn J Clin Oncol 2013; 3: 286-93. doi: 10.1093/jjco/hys232

59. Mannix KA, Blackburn IM, Garland A, Gracie J, Moorey S, Reid B, et al. Effectiveness of brief training in cognitive behaviour therapy techniques for palliative care practitioners. Palliat Med 2006;20:579-84. doi:10.1177/0269216306071058

60. Keall RM, Clayton JM, Butow PN. Therapeutic life review in palliative care: A systematic review of quantitative evaluations. J Pain Symptom Manage 2015;4:747-61. doi:10.1016/j.jpainsymman.2014.08.015 